\title{
Comparison of Pain Perception and Latency with Alkalinization of $2 \%$ Lidocaine Using $8.4 \%$ Sodium Bicarbonate: A Randomized Controlled Study
}

\author{
Vallala Pranitha ${ }^{1}$, Tapaswi Singh ${ }^{2}$, Kocherlakota S Dwijendra ${ }^{3}$, Gali Nagarjuna ${ }^{4}$, Zafaerah Sultana ${ }^{5}$, Karna Anusha $^{6}$
}

\begin{abstract}
Aims: Local anesthesia (LA) administration is a prerequisite for pain reduction but ironically becomes a source of pain and anxiety in children. The purpose of the study was to compare the latency and pain perception of alkalinized and nonalkalinized LA with adrenaline (1:80,000) by using $8.4 \%$ sodium bicarbonate.

Materials and methods: After obtaining the ethical clearance, 40 participants fulfilling the inclusion criteria were included and divided into two groups, namely, those who received alkalinized LA on the first appointment and those who received nonalkalinized LA 1 week later in the second appointment. Pain reaction on deposition of solution was quantified by visual analog scale (VAS).

Results: Statistical analysis was done using SPSS 25, Chi-square test for pain perception. An independent samples $t$ test was used to measure the latency time period. The mean latency time in alkalinized group was 118.9 seconds, while that for the nonalkalinized group was found to be 132.3 seconds, with a mean difference of 13.4 seconds between the two groups $(p=0.43)$.

Conclusion: Alkalinization hastens the onset of analgesia and reduces pain on injecting, making the alkalinized local anesthetic apt for cases with localized infection.

Clinical significance: Successful dental treatment outcomes demand efficient pain management which otherwise may lead to avoidance of dental care in children.

Keywords: Alkalinization, Inferior alveolar nerve block, Local anesthesia, Pain management, Sodium bicarbonate.

World Journal of Dentistry (2019): 10.5005/jp-journals-10015-1675
\end{abstract}

\section{INTRODUCTION}

The anticipation of pain associated with dental care is a significant deterrent to seeking treatment in children. ${ }^{1}$ Research had proved alkalinization of $L A$ with varying concentrations of $1.5-8.4 \%$ sodium bicarbonate is a potential method to increase the anesthetic success. ${ }^{2,3}$ Pain caused during administration of LA has been attributed to many factors, including the size, site and speed of injection, any localized inflammation, and low $\mathrm{pH}$ of the anesthetic solution.

The available local anesthetic solutions have low $\mathrm{pH}$, ranging from 3.5 to 5.5 and a shelf life of 3-4 years due to the addition of hydrochloric acid to these solutions. Thus, the administration of this acidic solution into the tissues leads to pain and burning sensation. ${ }^{4}$

Alkalinizing 2\% lidocaine with epinephrine and $8.4 \%$ sodium bicarbonate reduces the pain caused during administration and the latency of anesthesia by increasing the concentration of uncharged basic form, facilitates the penetration of lidocaine into the nerve cell, and also reduces postinjection tissue injury.

The aim of the study is to compare the pain perception and latency of alkalinized and nonalkalinized $2 \%$ lidocaine with adrenaline $(1: 80,000)$ by using $8.4 \%$ sodium bicarbonate.

\section{Materials and Methods}

This in vivo study was conducted in MNR Dental College, Department of Pedodontics and Preventive Dentistry, Sangareddy, Telangana, India, among 40 participants aged 7-9 years, with multiple treatment needs in the mandible and were requiring
${ }^{1,3-6}$ Department of Pedodontics and Preventive Dentistry, MNR Dental College and Hospital, Sangareddy, Telangana, India

${ }^{2}$ Department of Dental Surgery, Gandhi Medical College and Hospital, Hyderabad, Telangana, India

Corresponding Author: Vallala Pranitha, Department of Pedodontics and Preventive Dentistry, MNR Dental College and Hospital, Sangareddy, Telangana, India, Phone: +91 9440587228, e-mail: pranithavallala@gmail.com

How to cite this article: Pranitha V, Singh T, Dwijendra KS, et al. Comparison of Pain Perception and Latency with alkalinization of $2 \%$ Lidocaine Using 8.4\% Sodium Bicarbonate: A Randomized Controlled Study. World J Dent 2019;10(6):454-456.

Source of support: Nil

Conflict of interest: None

appointments. Before the start of the study, ethical clearance was taken and parents' consent was obtained from the Institution Ethical Committee and Research Board. The participants needed at least two clinical sessions requiring inferior alveolar nerve block (IANB). Children who are medically compromised and allergic to lignocaine were excluded from the study. In the first appointment, alkalinized LA was administered in the quadrant with dentoalveolar abscess and in the second appointment nonalkalinized LA was administered in the other quadrant.

The test areas were dried using a sterile gauze piece following adequate isolation. Lignocaine gel (Wocaine $2 \%$ gel) was applied to the test site with a cotton tip applicator in rubbing motion for 
30 seconds and left for 1 minute. Alkalinization was done at chair side at 1:10 ratio of $8.4 \%$ sodium bicarbonate and $2 \%$ lidocaine with epinephrine (1:80,000), e.g., 4 units of $8.4 \%$ sodium bicarbonate measured with 40 units of insulin syringe and $1 \mathrm{~mL}$ of lidocaine solution (LIGNOX $2 \%$ ).

Onset of anesthesia is checked by subjective symptoms and gingival probing after 30 seconds following IANB administration and then after 15 seconds until the patient reported absence of pain on probing.

The basic solution that is added has to be carefully mixed. ${ }^{5}$ If added in excess, then the $\mathrm{pH}$ rises and the noncharged basic form will precipitate out of solution, which can be seen as a white clouding of the solution that will adversely affect the efficacy of the local anesthetic solution. As the precipitation increases with time, alkalinized solutions should generally be freshly prepared and promptly used. The pain reaction on deposition of solution was quantified by VAS. ${ }^{6}$

\section{Statistical Analysis}

The sample size was selected. The Chi-square test was computed to calculate the association of the type of LA administered with pain perception of the participant. At the significance level of 0.05 and power of $90 \%$, the calculated sample size was 40 patients divided into alkalinized and nonalkalinized groups. Statistical analysis was done using SPSS 23. Continuous variables were analyzed using independent $t$ test to compare between the two groups. All proportions of both groups were compared using Chi-square test. $p<0.05$ was considered to be statistically significant.

\section{Results}

Chi-square test was computed to calculate the association of the type of LA administered with pain perception in children. Analysis revealed huge differences in the distribution of pain scores among alkalinized and nonalkalinized groups. In the alkalinized group, $70 \%$ of the participants responded with no pain as compared to only $5 \%$ in nonalkalinized group. Mild pain was recorded among $20 \%$ of the alkalinized group as compared to $55 \%$ of the nonalkalinized group. Moderate pain was experienced by only $5 \%$ of the participants in the alkalinized group as compared to $35 \%$ in the nonalkalinized group. Severe pain response was equally exhibited among both alkalinized and nonalkalinized group participants (5\%).

This analysis revealed a strong association of the pain response with nonalkalinized group as compared to no pain with the alkalinized group $(p<0.001)$.

An independent samples $t$ test was computed to compare the mean latency time period in the alkalinized and nonalkalinized LA groups.

Analysis showed that the mean latency time in alkalinized group was 118.9 seconds, while that in the nonalkalinized group was found to be 132.3 seconds. A difference of 13.4 seconds in the mean latency period was exhibited between the two groups ( $p=0.43$ ).

\section{Discussion}

Pain caused due to LA is not a mere sensation but is a complex consequential response of multifaceted interaction of physical, chemical, cognitive, psychological, behavioral, and social elements, which are conceptualized as stress and fear in children. ${ }^{7}$ Hence, significant concern of pediatric dentist is to manage the child with minimal discomfort. Behavior guidance and technique of administration of the local anesthetic are important considerations in the treatment process of pediatric patients.

Achieving profound anesthesia is the competence that the dental surgeons have to master. Pain during LA administration is due to low pH of 3.5 of the local anesthetic solution which is accentuated in the presence of inflammation or infected tissues with low $\mathrm{pH}$ and increased vasodilation, which alters the efficacy of anesthetic solution and behavior of the children which makes their management uncooperative. The potency of the anesthetics is achieved by increasing the availability of deionized molecules and reducing the concentration of hydrogen ions by alkalinizing the anesthetic solution. ${ }^{8}$

The present study was undertaken to compare the pain perception and latency of alkalinized and nonalkalinized LA among 40 participants of age-groups 7-9 years. Patients presenting to the dental emergency clinic with facial swelling were examined for verification of the odontogenic nature of their swelling and evaluated for the presence and extent of clinical swelling without actively draining sinus in mandible. In the first appointment, alkalinized LA was administered; and in the second appointment after 1 week, non-alkalinized LA was administered for extraction on the contralateral side.

The appropriate proportion and concentration of drug and the amount in the mixture is still controversial. The solution of $2 \%$ lidocaine with epinephrine 1:80,000 was freshly prepared at chair side by mixing with $8.4 \%$ sodium bicarbonate in the ratio of 10:1. Varying concentrations of $\mathrm{NaHCO}_{3}{ }^{9}$ such as $0.1 \mathrm{~mL}$ of $8.4 \%$ with $1.7 \mathrm{~mL}$ of $2 \%$ lidocaine with epinephrine $(1: 80,000)$ administered as maxillary infiltration in periapical surgeries of maxillary anterior teeth reported no pain or mild pain among the participants. ${ }^{10}$ Similar results were noted with epinephrine mixed with $7.5 \%$ $\mathrm{NaHCO}_{3}$ at a ratio of 10:0.5 in adult patients who had dentoalveolar abscess and found IANB was effective in producing efficient anesthesia even in infection sites, proving that it neutralizes the acidic environment. The freshly prepared alkalinized solution was then injected on the side of quadrant with dentoalveolar abscess in first appointment within 5 minutes of its preparation, while the other quadrant received lidocaine hydrochloride with adrenaline. Visual and tactile examinations were performed before the procedure. Following injection, pain at the site of injection was assessed from subjective symptoms and gingival probing 30 seconds after injection followed by 15 seconds until the patient reported absence of pain on probing.

Patient was asked to describe the pain caused by the delivery of anesthetic solution on a VAS, which is a 10-point horizontal scale with marking $0 \mathrm{~mm}=$ no pain; $1-3 \mathrm{~mm}=$ mild pain, i.e., pain reported in response to questioning and without any behavioral signs; $4-6 \mathrm{~mm}=$ moderate pain, i.e., pain reported spontaneously without questioning; and $7-10 \mathrm{~mm}=$ severe pain, i.e., strong vocal response or response accompanied by withdrawal of arms or tears. The selected VAS is numerical and pictorial based, which is easy to code by the children and prevents false-positive results $(p<0.001)$.

Among the 40 participants who were administered alkalinized LA, 70\% had responded with no pain as compared to $5 \%$ from nonalkalinized group. This result demonstrates that alkalinized LA provides more comfort on injection compared to nonalkalinized solution. Studies conducted by Al-Sultan et al., Kashyap et al., Schellenberg, and Saatchi et al..$^{9,11-13}$ had shown success results, demonstrating no or less pain with alkalinization, whereas studies 
by Chopra et al., Whitcomb et al., and Hobeich et al. ${ }^{4,14,15}$ showed low success in pain reduction.

The latency of anesthesia was assessed by subjective and objective signs, which were checked with a stopwatch and the time was recorded in seconds. Patients were asked to note down the time of pain sensation from delivering of the solution and postextraction to assess the duration of anesthesia. Analysis showed that the mean latency time in the alkalinized group was 118.9 seconds, while that in the nonalkalinized group was found to be 132.3 seconds $(p=0.43)$.

The studies conducted by Kashyap et al., Sinnott et al., and Malamed et al. ${ }^{11,16,17}$ showed reduced latency with alkalinized anesthetic solution, whereas decrease in latency with alkalinizing could not be demonstrated by Whitcomb et al., Hobeich et al., and Chow et al. ${ }^{14,15,18}$

Alkalinizing lidocaine solution with sodium bicarbonate not only raises the $\mathrm{pH}$ of the solutions but also leads to the production of carbon dioxide $\left(\mathrm{CO}_{2}\right)$ and water as a by-product when interacted with hydrochloric acid in $\mathrm{LA} . \mathrm{CO}_{2}$ has a direct depressant effect on axon. ${ }^{19}$ Limitation of the present study was smaller sample size and shelf life of the solution is less.

\section{Conclusion}

Painless infiltration and block may be achievable in cases of localized infections, with the addition of sodium bicarbonate in local anesthetics. The reduction in time to onset of action and an increase in the potency of anesthesia are the major advantages of alkalinization of local anesthetic solutions. Further studies and analysis of the same with larger sample sizes should be done to prove the efficacy of alkalinization to incorporate the procedure in routine clinical pediatric dentistry.

\section{References}

1. Comerci AW, Maller SC, Townsend RD, et al. Effect of a new local anesthetic buffering device on pain reduction during nerve block injections. Gen Dent 2015;63(6):74-78.

2. Afolabi O, Murphy A, Chung B, et al. The effect of buffering on pain and duration of local anesthetic in the face: a double-blind, randomized controlled trial. Can J Plast Surg 2013;21(4):209-212.

3. Kraut JA, Madias NE, et al. Intravenous sodium bicarbonate in treating patients with severe metabolic acidemia. AJKD 2019;73(4):572-575. DOI: 10.1053/j.ajkd.2018.08.011.

4. Chopra R, Jindal G, Sachdev V, et al. Double-blind crossover study to compare pain experience during inferior alveolar nerve block administration using buffered two percent lidocaine in children. Pediatr Dent 2016;38(1):25-29.

5. Brandis K. Alkalinization of local anesthetic solutions. Aust Prescr 2011;34(6):173-175. DOI: 10.18773/austprescr.2011.091.

6. Kumar Bapna PA, Mane PN, Ganiger CR, et al. A survey of perception of pain and discomfort with elastomeric separators in patients undergoing orthodontic treatment in Western Maharashtra region. J Oral Res Rev 2017;9:56-61. DOI: 10.4103/jorr.jorr_42_16.

7. Casamassimo PS, McTigue DJ, Fields HW, et al. Text Book of Pediatric Dentistry: Infancy through adolescence, ch. 6 5th ed., Elsevier Inc; 2013.

8. Catchlove RFH. The influence of $\mathrm{CO}_{2}$ and pH on local anesthetic action. J Pharmacol Exp Therap 1972;181(2):298-309.

9. Al-Sultan FA, Fathie WK, Hamid RS. A clinical evaluation on the alkalization of local anesthetic solution in periapical surgery. Al-Rafidain Dent J 2006;6(1):71-77.

10. Lingaraj JB, Vijayakumar A. A randomized control study to compare the efficacy of carbonated lignocaine with lignocaine hydrochloride in mandibular nerve blocks: a pilot study. IOSR Journal of Dental and Medical Sciences (IOSR-JDMS) 2018;17:13-16.

11. Kashyap VM, Desai R, Reddy PB, et al. Effect of alkalinisation of lignocaine for intraoral nerve block on pain during injection, and speed of onset of anaesthesia. Br J Oral Maxillofac Surg 2011;49(8):e72-e75. DOI: 10.1016/j.bjoms.2011.04.068.

12. Schellenberg J, Drum M, Reader A, et al. Effect of buffered $4 \%$ lidocaine on the success of the inferior alveolar nerve block in patients with symptomatic irreversible pulpitis: a prospective, randomized, double blind study. J Endod 2015;41(6):791-796. DOI: 10.1016/ j.joen.2015.02.022.

13. Saatchi M, Farhad AR, Shenasa N, et al. Effect of sodium bicarbonate buccal infiltration on the success of inferior alveolar nerve block in mandibular first molars with symptomatic irreversible pulpitis: a prospective, randomized double-blind study. J Endod 2016;42(10):1458-1461. DOI: 10.1016/j.joen.2016.07.004.

14. Whitcomb M, Drum M, Reader A, et al. A prospective, randomized, double-blind study of the anesthetic efficacy of sodium bicarbonate buffered 2\% lidocaine with 1:100,000 epinephrine in inferior alveolar nerve blocks. Anesth Prog 2010;57(2):59-66. DOI: 10.2344/00033006-57.2.59.

15. Hobeich P, Simon S, Schneiderman E, et al. A prospective, randomized, double-blind comparison of the injection pain and anesthetic onset of $2 \%$ lidocaine with 1:100,000 epinephrine buffered with $5 \%$ and $10 \%$ sodium bicarbonate in maxillary infiltrations. J Endod 2013;39(5):597-599. DOI: 10.1016/j.joen.2013.01.008.

16. Sinnott CJ, Garfield JM, Thalhammer JG, et al. Adidition of sodium bicarbnate to lidocaine decreases the duration of peripheral nerve block in the rat. Anesthesiology 2000;93(4):1045-1052. DOI: 10.1097/00000542-200010000-00028.

17. Malamed SF, Tavana S, Falkel M. Faster onset and more comfortable injection with alkalinized $2 \%$ lidocaine with epinephrine 1:100,000. Compendium of Continuing Education in Dentistry (Jamesburg, NJ: 1995) 2013;34(Spec No 1):10-20.

18. Chow MY, Sia AT, Koay CK, et al. Alkalinization of lidocaine does not hasten the onset of axillary brachial plexus block. Anesth Analg 1998;86(3):566-568. DOI: 10.1213/00000539-19980300000024.

19. Catchlove RF. The influence of $\mathrm{CO}_{2}$ and $\mathrm{pH}$ on local anesthetic action. Survey of Anesthesiology 1973;17(2):184-185. DOI: 10.1097/00132586$197304000-00053$. 